OPEN ACCESS

Edited by:

Guo Chen,

Jinan University, China

Reviewed by:

Liang Xu,

National University of

Singapore, Singapore

Naidi Yang,

Nanjing Tech University, China

*Correspondence:

Shengzhang Lin

1311046@zju.edu.cn

Shusen Zheng

shusenzheng@zju.edu.cn

†These authors share first authorship

Specialty section:

This article was submitted to

Precision Medicine,

a section of the journal

Frontiers in Medicine

Received: 09 July 2020

Accepted: 04 August 2020

Published: 16 September 2020

Citation:

Zhang L, Qiao Y, Huang J, Wan D, Zhou L, Lin S and Zheng S (2020)

Expression Pattern and Prognostic Value of Key Regulators for m6A RNA

Modification in Hepatocellular

Carcinoma. Front. Med. 7:556.

doi: 10.3389/fmed.2020.00556

\section{Expression Pattern and Prognostic Value of Key Regulators for m6A RNA Modification in Hepatocellular Carcinoma}

\author{
Lele Zhang ${ }^{1,2,3,4,5 t}$, Yiting Qiao ${ }^{1,3,4,5+}$, Jiacheng Huang ${ }^{1,2,3,4,5}$, Dalong Wan ${ }^{1}$, Lin Zhou ${ }^{1,3,4,5}$, \\ Shengzhang Lin ${ }^{2,6 *}$ and Shusen Zheng ${ }^{1,3,4,5,6 *}$
}

${ }^{1}$ The First Affiliated Hospital, Zhejiang University School of Medicine, Division of Hepatobiliary and Pancreatic Surgery, Department of Surgery, Hangzhou, China, ${ }^{2}$ School of Medicine, Zhejiang University, Hangzhou, China, ${ }^{3}$ NHC Key Laboratory of Combined Multi-organ Transplantation, Hangzhou, China, ${ }^{4}$ Key Laboratory of the Diagnosis and Treatment of Organ Transplantation, Research Unit of Collaborative Diagnosis and Treatment for Hepatobiliary and Pancreatic Cancer, Chinese Academy of Medical Sciences (2019RU019), Hangzhou, China, ${ }^{5}$ Key Laboratory of Organ Transplantation, Hangzhou, China, ${ }^{6}$ Shulan (Hangzhou) Hospital Affiliated to Zhejiang Shuren University Shulan International Medical College, Hangzhou, China

As the most prevalent type of mRNA modification in mammals, N6-methyladenosine $(\mathrm{m} 6 \mathrm{~A})$ is involved in various biological processes. Accumulating studies have indicated that the deregulation of m6A RNA modification is linked to cancer and other diseases. However, its implications in hepatocellular carcinoma (HCC) remain poorly characterized. Herein, we sought to investigate the expression pattern of 13 key regulators for m6A RNA modification and to evaluate their prognostic value in HCC. First, we systematically analyzed data from The Cancer Genome Atlas (TCGA) database pertaining to patient clinical information and mRNA gene expression data. We found that 11 out of 13 key regulators for m6A RNA modification showed significantly higher expression levels in HCC. Subsequently, we identified two subgroups (clusters 1 and 2) via consensus clustering based on the expression of 13 m6A RNA methylation regulators. Cluster 2 had a worse prognosis and was also significantly correlated with higher histological grade and pathological stage when compared with cluster 1. Moreover, cluster 2 was remarkedly enriched for cancer-related pathways. We further constructed a robust risk signature of five regulators for m6A RNA modification. Further analysis indicated that this risk signature could be an independent prognostic factor for HCC, and the prognostic relevance of this five-gene risk signature was successfully validated using the Gene Expression Omnibus (GEO) dataset. Finally, we established a novel prognostic nomogram on the basis of age, gender, histological grade, pathological stage, and risk score to precisely predict the prognosis of patients with HCC. In summary, we herein uncovered the vital role of regulators for m6A RNA modification in HCC and developed a risk signature as a promising prognostic marker in $\mathrm{HCC}$ patients.

Keywords: m6A, hepatocellular carcinoma, bioinformatics, prognostic signature, nomogram 


\section{INTRODUCTION}

Hepatocellular carcinoma (HCC) remains among the most prevalent and deadly types of cancer worldwide, with more than 700,000 deaths documented annually (1). Epidemiological studies have shown that hepatitis virus infection, alcohol abuse, and aflatoxin contamination are primary risk factors for HCC (2). Due to the lack of apparent symptoms at the early stages of HCC, the majority of patients diagnosed with this cancer are first identified in an advanced stage where the complication of intrahepatic and/or extrahepatic metastasis has taken place (3). Even though the prognosis for HCC patients has improved due to recent advances in various treatment approaches, including surgical tumor resection, targeted drug therapy, transarterial chemoembolization, and liver transplantation, the 5 -year survival rate remains dismal due to the high rate of metastasis and recurrence (4). At present, the tumor, lymph node, and metastasis (TNM) staging system is still the most widely adopted prognostic indicator for monitoring the progress of HCC. However, HCC is highly heterogeneous; therefore, patients with the same TNM stage often present remarkable differences in survival outcomes and treatment responses. Therefore, to improve the unsatisfactory outcomes of patients with HCC, it is important to identify novel and reliable molecular signatures for prognostic prediction.

Methylation at the position N6 of adenosine, also known as N6-methyladenosine (m6A) modification, is evolutionarily conserved and widely present in most eukaryotic species (yeast, plants, and mammals) and viral mRNA $(5,6)$. The process of m6A modification is reversible and dynamic and is controlled by methyltransferases ("writers"), demethylases ("erasers"), and methyl-binding proteins ("readers") (7). Methyltransferases, such as METTL3, RBM15, KIAA1429, METTL14, WTAP, and $\mathrm{ZC} 3 \mathrm{H} 13$ are responsible for the methylation modification of RNA (8). Demethylases, including ALKBH5 and FTO, mediate the process of demethylation of RNA $(9,10)$. Methylbinding proteins, including YTHDF1, HNRNPC, YTHDC1, YTHDF2, and YTHDC2, can recognize m6A-modified sites and preferentially bind to such sites to regulate downstream signals (11). RNA m6A modification is involved in many vital cellular processes, such as gene expression, alternative splicing, degradation, translation of mRNA, and RNA-protein interaction (12).

The poor prognosis of cancer patients is due to the unique malignant biological characteristics of the cancer, including epithelial-mesenchymal transition (EMT), cancer stem cell formation, signaling transduction, tumor angiogenesis, and cancer metabolism. Several studies have shown that aberrant m6A RNA modification plays key roles in these biological processes closely associated with the HCC progression by regulating mRNA stability or protein translation (13-15). For example, METTL3- and YTHDF1-dependent m6A modification could promote EMT by enhancing the translation of Snail mRNA in liver cancer (16). In hypoxic environment, the expression of ALKBH5 was stimulated in a HIF-dependent manner in breast cancer. Overexpression of ALKBH5 decreased m6A modification and stabilizes NANOG mRNA, thus resulting in higher stemness
(17). Considering that hypoxia also plays an important role in the progression of $\mathrm{HCC}, \mathrm{m} 6 \mathrm{~A}$ may also promote the formation of cancer stem cells through a similar approach. As research continues, m6A modification has been shown to participate in the many signaling pathways, including but not limited to the MYC/CEBPA, Wnt/PI3K-Akt, AFF4/NF- $\kappa$ B/MYC, YAP, and TGF- $\beta$ signaling pathways, to promote cancerous growth as well as angiogenesis $(18,19)$. In addition, m6A modification can modulate cancer metabolism through downregulating the translation of ATG5/7, the key signal node for autophagy, as well as upregulating the translation of 6PGD, the central player of pentose phosphate pathway $(20,21)$. To summarize, deregulation of m6A modification profoundly promoted the malignancy of cancer. This explains why m6A RNA modification has prognostic impacts on patients with HCC.

In this study, transcriptome data from The Cancer Genome Atlas (TCGA) datasets were used to assess the expression of 13 key regulators for m6A RNA modification in HCC. Additionally, HCC patients were categorized into two clusters according to the expression pattern of regulators for $\mathrm{m} 6 \mathrm{~A}$ RNA modification by consensus clustering, and two clusters exhibited significantly different clinical outcomes. Furthermore, a risk signature prognostic prediction model was established and showed a favorable predictive value for HCC patients. More importantly, the prognostic relevance of this risk signature was successfully validated in the Gene Expression Omnibus (GEO) database.

\section{MATERIALS AND METHODS}

\section{Data Collection}

RNA-sequencing transcriptomic data and corresponding clinical information for patients with HCC were obtained from TCGA (https://portal.gdc.cancer.gov/; until February 21, 2020). A total of 374 HCC cases and 50 normal adjacent tumor tissues were included for further analysis.

Thirteen currently known genes, including YTHDF1, YTHDF2, YTHDC1, YTHDC2, METTL3, METTL14, ALKBH5, FTO, HNRNPC, KIAA1429, RBM15, WTAP, and ZC3H13, are recognized as $\mathrm{m} 6 \mathrm{~A}$ methylation regulators. The expression data of these 13 genes were extracted for subsequent analysis from the HCC cohort of the TCGA database. For external validation, we used an independent cohort (GSE54236) containing 78 HCC samples with corresponding gene expression data and the survival information, which were obtained from the GEO (http://www.ncbi.nlm.nih.gov/geo).

\section{Bioinformatics Analysis}

Differentially expressed genes encoding m6A RNA methylation regulators between HCC and normal tissues were screened using the Wilcoxon test method in $\mathrm{R}$ (version $\mathrm{R}$ 3.6.3, https:// www.r-project.org/). Significance criteria were as follows: false discovery rate $(\mathrm{FDR})<0.05$ and absolute $\log 2$ fold change (FC) $>1$. Subsequently, a vioplot was used to exhibit the expression of these m6A-related genes in 374 HCC patients and 50 normal adjacent samples. Spearman correlation analyses were 
conducted using $\mathrm{R}$ to identify the association between m6A RNA methylation regulators.

To assess the link between m6A RNA methylation regulators expression and HCC prognosis, HCC cohort was clustered into two different subgroups using the "ConsensusClusterPlus" $\mathrm{R}$ package. Principal component analysis (PCA) was then carried out using the "ggplot2" and "limma" package to verify the results of the classification. A survival curve was plotted to compare survival between subgroups based on the KaplanMeier analysis log-rank test. The difference in clinical parameters between the two clusters was determined using the Chi-square test. In order to conduct functional annotation of the genes with different expression in two subgroups, we performed Gene ontology (GO) and Kyoto Encyclopedia of Genes and Genomes (KEGG) analyses.

Univariate Cox regression analyses were utilized to assess the relationship between m6A-related genes and overall survival (OS). Subsequently, to avoid overfitting, we performed least absolute shrinkage and selection operator (LASSO) Cox regression to eliminate the highly correlated genes with the "glment" package. Ultimately, a five-m6A-regulatory-gene risk signature was identified. To generate a risk score, we multiplied

A

L

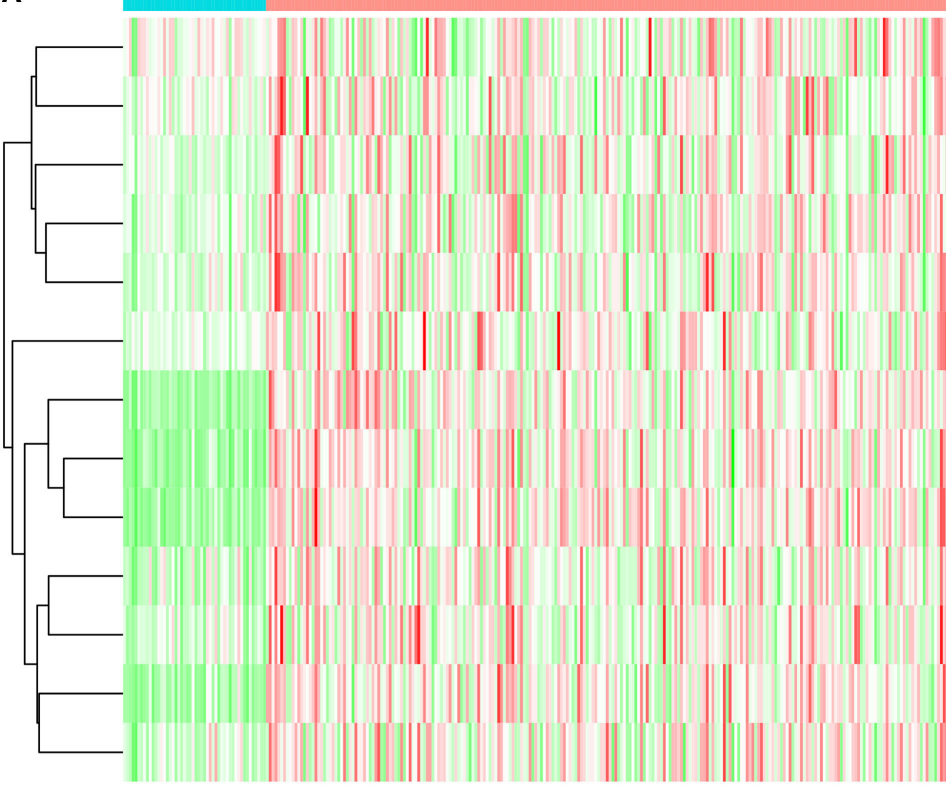

B

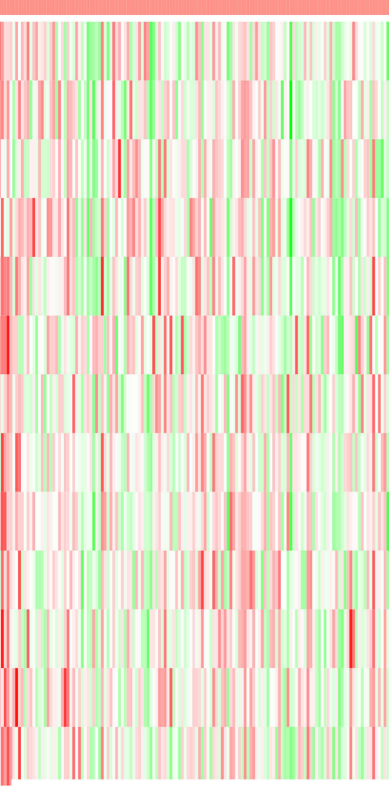

Type

ZC3H13

METTL14

$\mathrm{FTO}^{* * *}$

0

YTHDC2***

YTHDC1***

ALKBH5***

KIAA1429***

HNRNPC ${ }^{* * *}$

$\mathrm{METTL}^{* * *}$

YTHDF2 $2^{* * *}$

RBM15***

YTHDF1 $1^{* * *}$

WTAP ${ }^{* * *}$

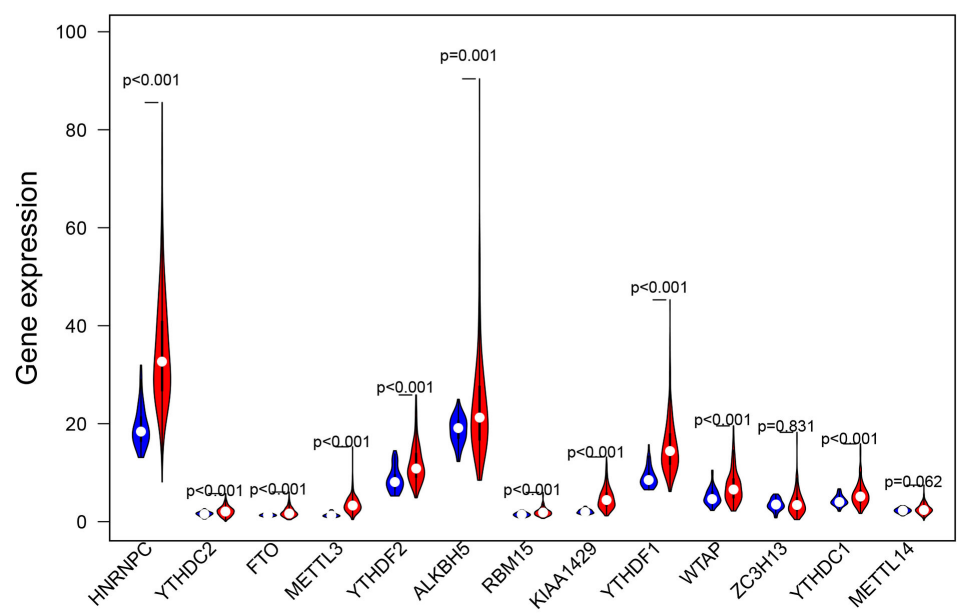

C

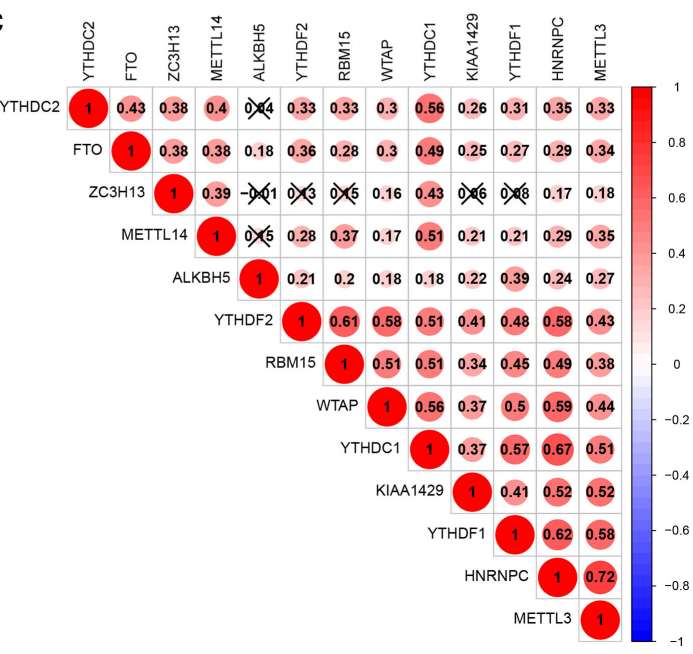

FIGURE 1 | Expression of m6A modification regulators in HCC. (A) The heatmap visualizes the expression levels of m6A RNA modification regulators in each sample. "N" represents normal samples and "T" represents tumor samples. Green represents low expression and red represent high expression. (B) The vioplot shows the differentially m6A RNA modification regulators in HCC. Blue represents normal sample and red represents HCC sample. White spot represents the median value of expression. (C) Spearman correlation analysis of the 13 m6A RNA modification regulators in $\mathrm{HCC} .{ }^{\star \star \star} p<0.001$. 
the gene expression and its coefficient obtained from the LASSO Cox regression. Median risk scores were then used to separate HCC patients into low- and high-risk groups. Kaplan-Meier analysis was performed using "survival" package. Receiver operating characteristic (ROC) curves were used to examine the accuracy of the model for prognostic prediction. The differences in clinicopathological variables between low- and high-risk groups were assessed via Chi-square text and visualized via a heatmap. In addition, univariate and multivariate Cox regression analyses were used as a means of assessing whether the risk score was an independent prognostic indicator.

To validate the prognostic value of this five-m6A-regulatorygene risk signature, we used GSE54236 datasets as the validation cohort. Patient risk scores were calculated using the same formula as above. We applied the same cutoff criteria to classify the patients into low- and high-risk groups. Subsequently, KaplanMeier survival analysis and ROC curve analysis were performed to assess the prognostic value.
A

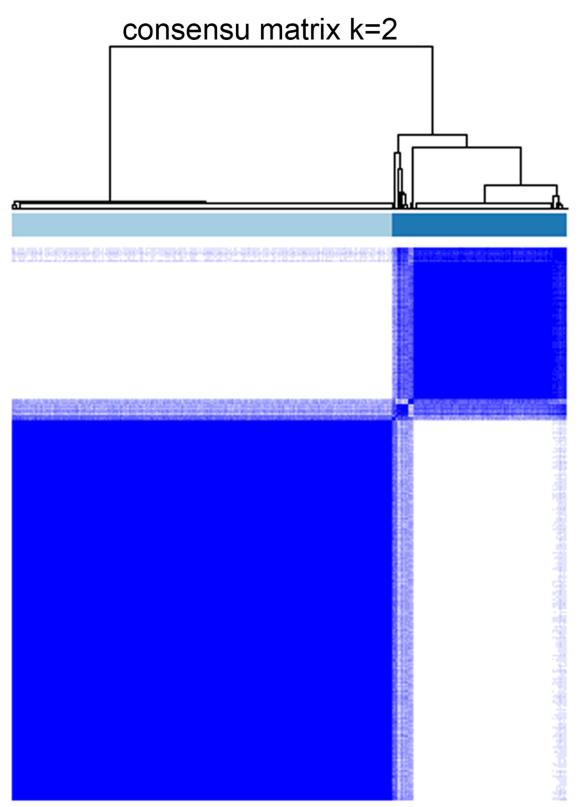

C
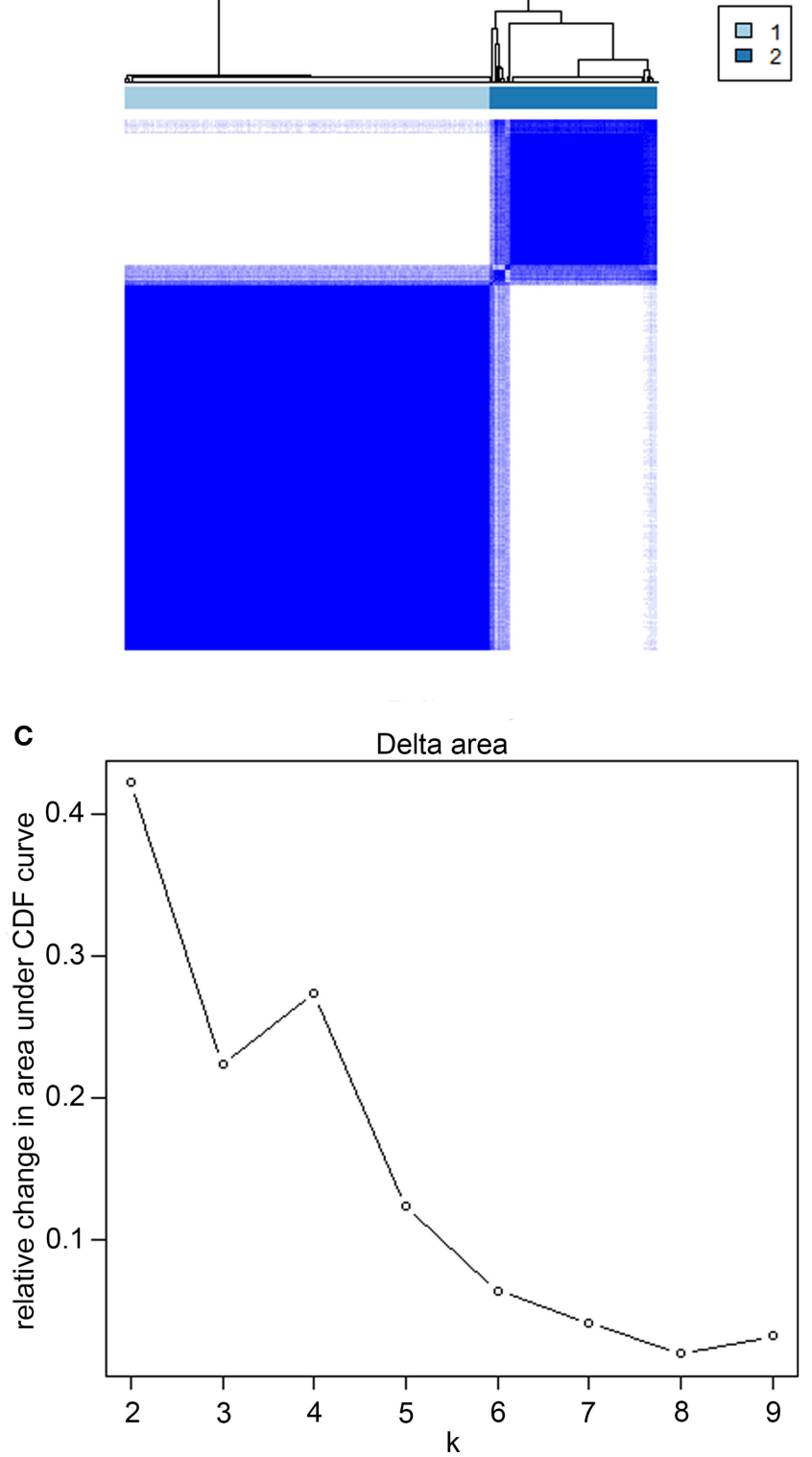

B

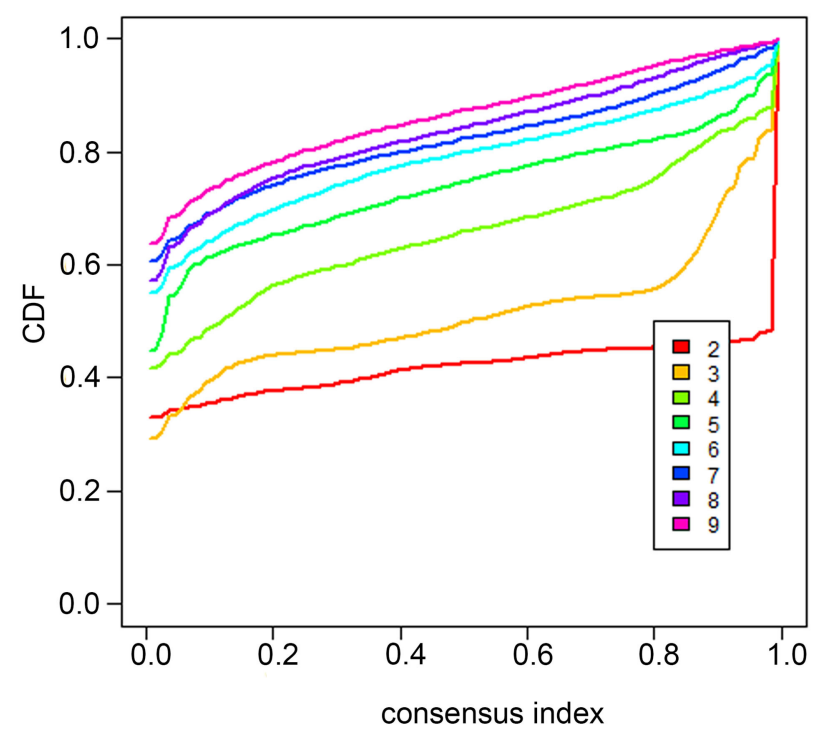

D

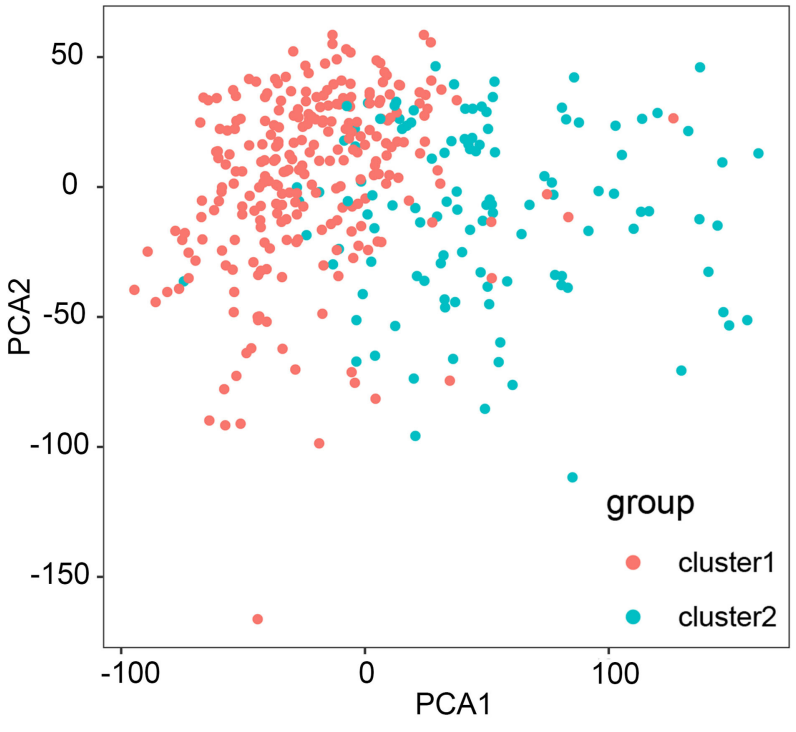

FIGURE 2 | Consistent cluster analysis of HCC. (A) The correlation between subgroups when cluster numbers $k=2$. (B) Cumulative distribution function (CDF) is displayed for $k=2-9$. (C) The relative change in area under the CDF curve for $k=2-9$. (D) Principal component analysis of the RNA-seq data. Red dots represent cluster 1 and cyan dots represent cluster 2. 
Finally, clinical factors (gender, age, histologic grade, and pathological stage) and risk score were utilized to develop a prognostic nomogram to predict 1-, 3-, and 5-year survival of patients with HCC via "rms" package.

All R packages mentioned above were obtained from http:// www.bioconductor.org.

\section{Statistical Analysis}

$\mathrm{R}$ software (version 3.6.3) was utilized for all statistical analyses, and $p<0.05$ was the significance threshold.

\section{RESULTS}

\section{Identification of Differentially Expressed m6A RNA Modification Regulators in HCC}

We conducted a differential expression analysis of $13 \mathrm{~m} 6 \mathrm{~A}$ regulatory genes in HCC $(n=375)$ and adjacent tissues ( $n$ $=50$ ). Heatmap clearly revealed that most of these m6Arelated genes were differentially expressed between HCC and control tissues (Figure 1A). Specifically, the expression levels of HNRNPC, YTHDF2, FTO, METTL3, YTHDF2, ALKBH5, RBM15, KIAA1429, YTHDF1, WTAP, and YTHDC1 (all $p<$ 0.001 ) were remarkably higher in tumor samples than those in normal tissues. There was no significant difference for ZC3H13 $(p=0.831)$ and METTL14 $(p=0.062)$ (Figure 1B). Moreover, a correlation analysis was performed to further understand the intrinsic association between 13 m6A RNA modification regulators. Figure 1C shows that the correlation between METTL13 and HNRNPC is the most significant. The HNRNPC expression level is most likely to be positively correlated with METTL13.

\section{Use of Consensus Clustering Based on m6A RNA Modification Regulators to Identify Two HCC Patient Clusters With Distinct Clinical Outcomes}

To further investigate the clinical relevance of $13 \mathrm{~m} 6 \mathrm{~A}$ RNA modification regulators, we clustered HCC patients into subgroups according to their gene expression patterns.

Based on similarities in m6A RNA modification regulators, $k=2$ gave the optimum clustering and the HCC cohort could be divided into two distinct and non-overlapping clusters (Figures 2A-C). In order to verify the result of the clustering, we further analyzed these two clusters by PCA. The PCA plot showed significant distinction between cluster 1 and cluster 2 (Figure 2D). We then assessed whether there were significant differences in OS and clinical parameters between these two clusters. As a result, a significantly better OS was observed in cluster 1, compared to that in cluster $2(p<0.01)$ (Figure 3B). Moreover, the expression level of most m6A RNA modification regulators of cluster 2 was higher than that of cluster 1 (Figure 3A). Compared with cluster 1, cluster 2 was significantly associated with female gender $(p<0.05)$, higher histologic grade $(p<0.001)$, and higher pathological stage $(p<0.05)$. No significant difference was observed for age (Figure 3A). Thus, the results of consensus clustering suggested a close association between the expression pattern of m6A RNA modification regulators and HCC malignancy.

In order to further interpret the clustering results from the perspective of fundamental biological processes, we performed GO and KEGG analyses on genes that are differentially expressed between cluster 1 and cluster 2. According to the results of the GO analysis, upregulated genes were primarily enriched in malignancy-related biological processes, such as "humoral
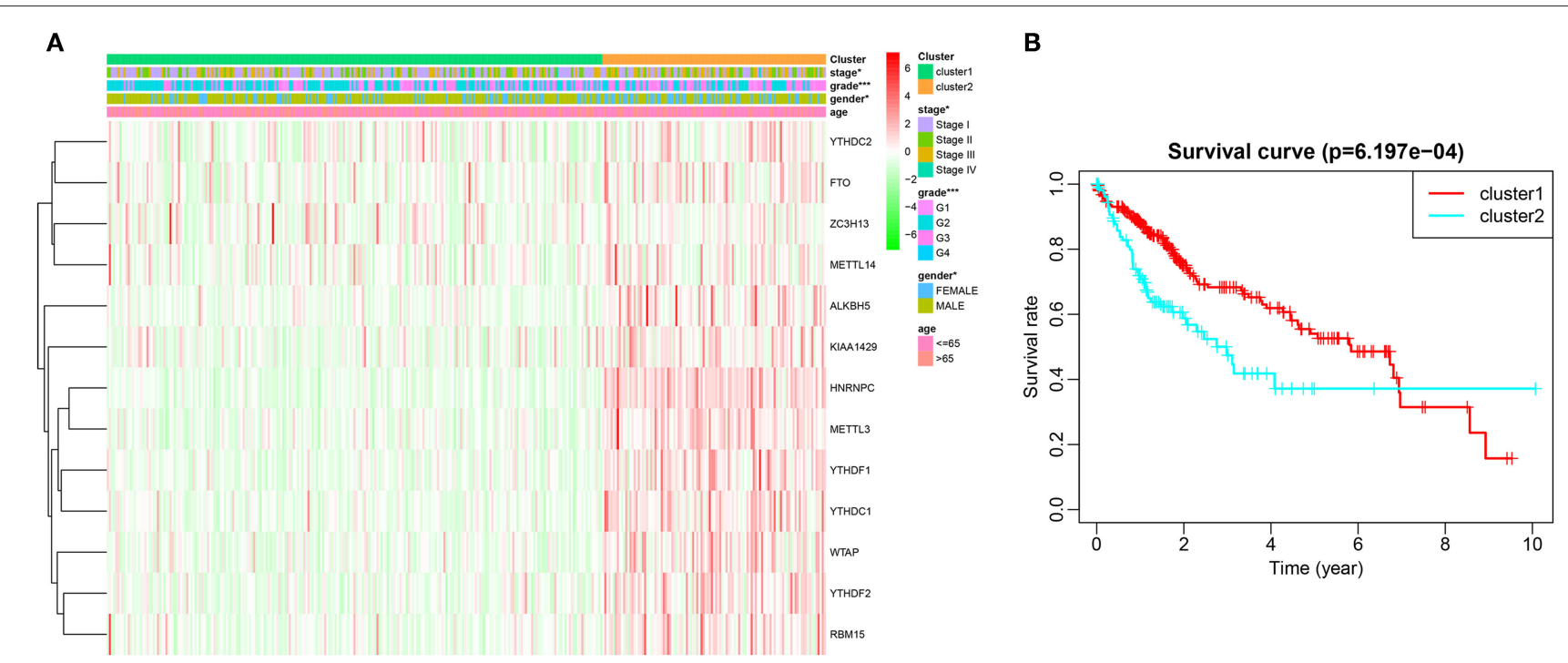

FIGURE 3 | Difference in clinicopathological features and overall survival between cluster 1 and cluster 2. (A) Heatmap and clinicopathological characteristics of these two clusters. Green represents low expression and red represent high expression. (B) Comparison of overall survival (OS) between cluster 1 and cluster $2 .{ }^{*} p<0.05$, ${ }^{* * *} p<0.001$. 
immune response mediated by circulating immunoglobulin," "B cell mediated immunity," "immunoglobulin mediated immune response," "complement activation, classical pathway," and "protein activation cascade" (Figures 4A,B). The results of the KEGG analysis showed that these upregulated genes were significantly enriched in "cell cycle," "herpes simplex virus 1 infection," and "extracellular matrix (ECM)-receptor interaction" (Figures 4C,D).

\section{Establishment of a Prognostic Risk Model Based on the Expression Level of m6A Regulator Genes}

Considering the strong association between m6A RNA methylation regulators and the prognosis of HCC patients, we applied a univariate Cox regression analysis on the expression levels of 13 key regulators. The results showed that nine out of 13 regulators were significantly correlated with OS $(p<0.05)$
(Figure 5A). Among these nine regulators, YTHDF1, YTHDF2, METTL3, KIAA1429, HNRNPC, WTAP, YTHDC1, and RBM15 were considered as risky genes, with $\mathrm{HR}>1$; meanwhile only ZC3H13 was considered as a protective gene, with $\mathrm{HR}<1$. Subsequently, LASSO Cox regression analysis was used to identify the m6A RNA modification regulators with the strongest prognostic power (Figures 5B,C). Ultimately, five optimal genes (YTHDF1, YTDFH2, METTL3, KIAA1429, and ZC3H13) were selected for the establishment of the risk model for HCC, and the corresponding coefficients from the LASSO algorithm (Figure 5D). The formula for calculating the risk score was as follows: risk score $=(0.084 *$ expression value of YTHDF2 $)+$ $(0.025 *$ expression value of YTHDF 1$)+(0.101 *$ expression value of METTL3) + $(0.046 *$ expression value of KIAA1429) $(0.107 *$ expression value of $\mathrm{ZC} 3 \mathrm{H} 13)$.

To explore the prognostic role of this five-gene signature model, HCC patients were separated into low- and highrisk groups based on the median risk score. Survival analysis
A

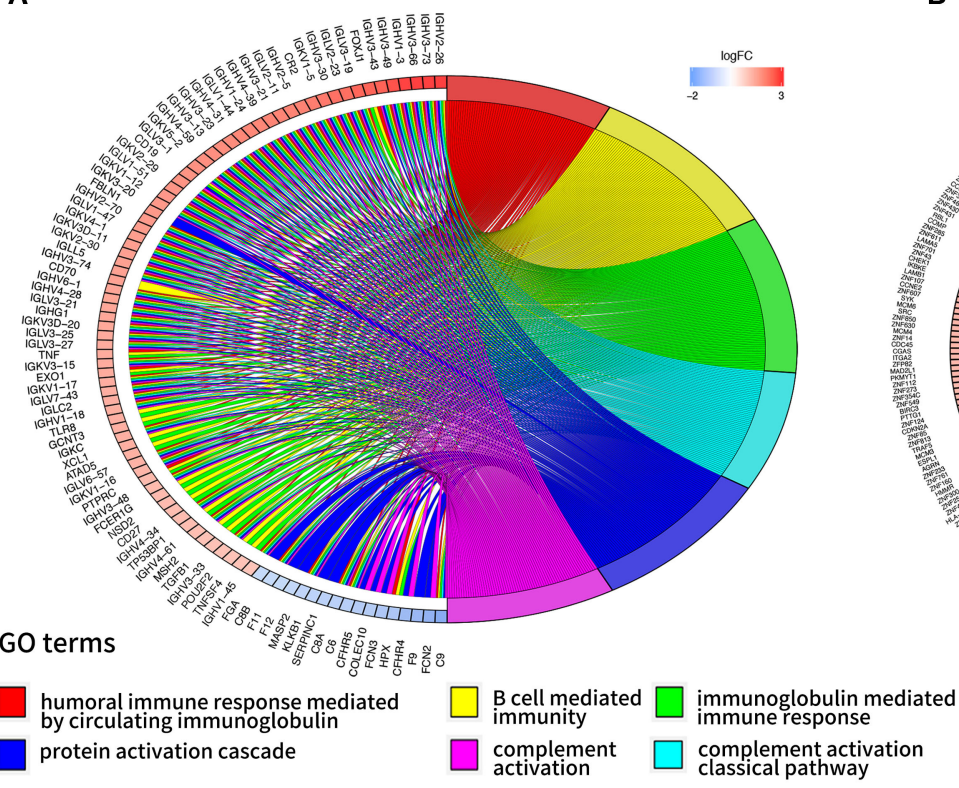

C

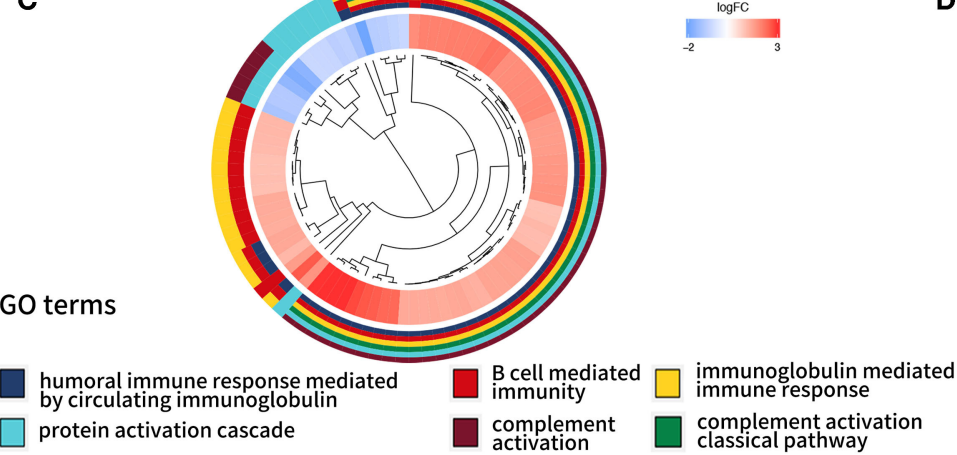

B

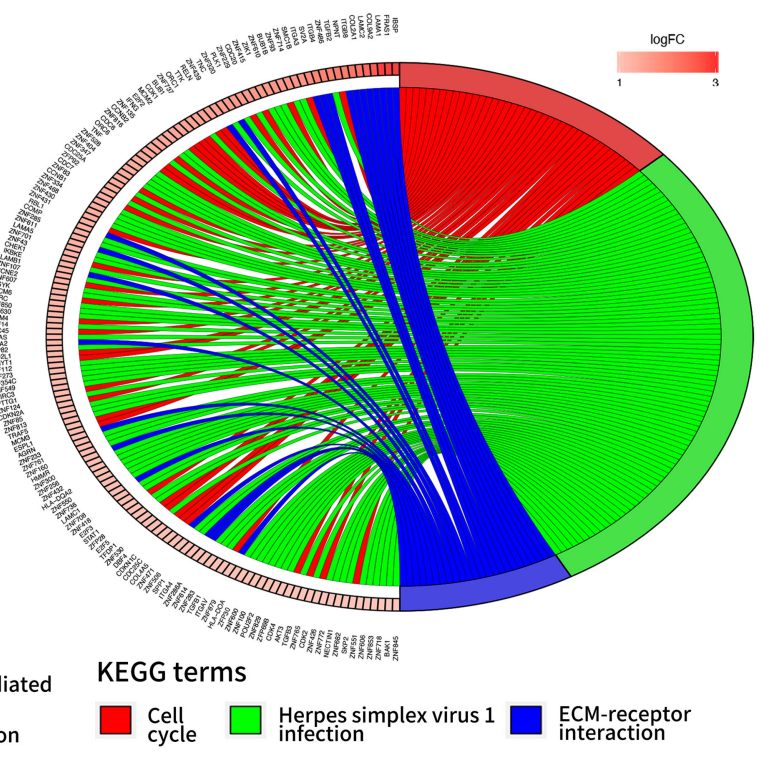

D

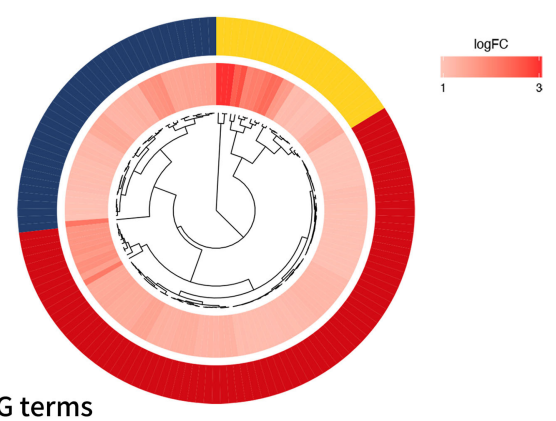

KEGG terms

Cell
cycle

Herpes simplex virus 1 ECM-receptor
interaction

FIGURE 4 | Gene ontology (GO) and Kyoto Encyclopedia of Genes and Genomes (KEEG) analyses of differentially expressed genes between two clusters. Function annotation on higher expressed genes in cluster 2 using $\mathrm{GO}$ terms (A,B) and KEGG pathway (C,D). 

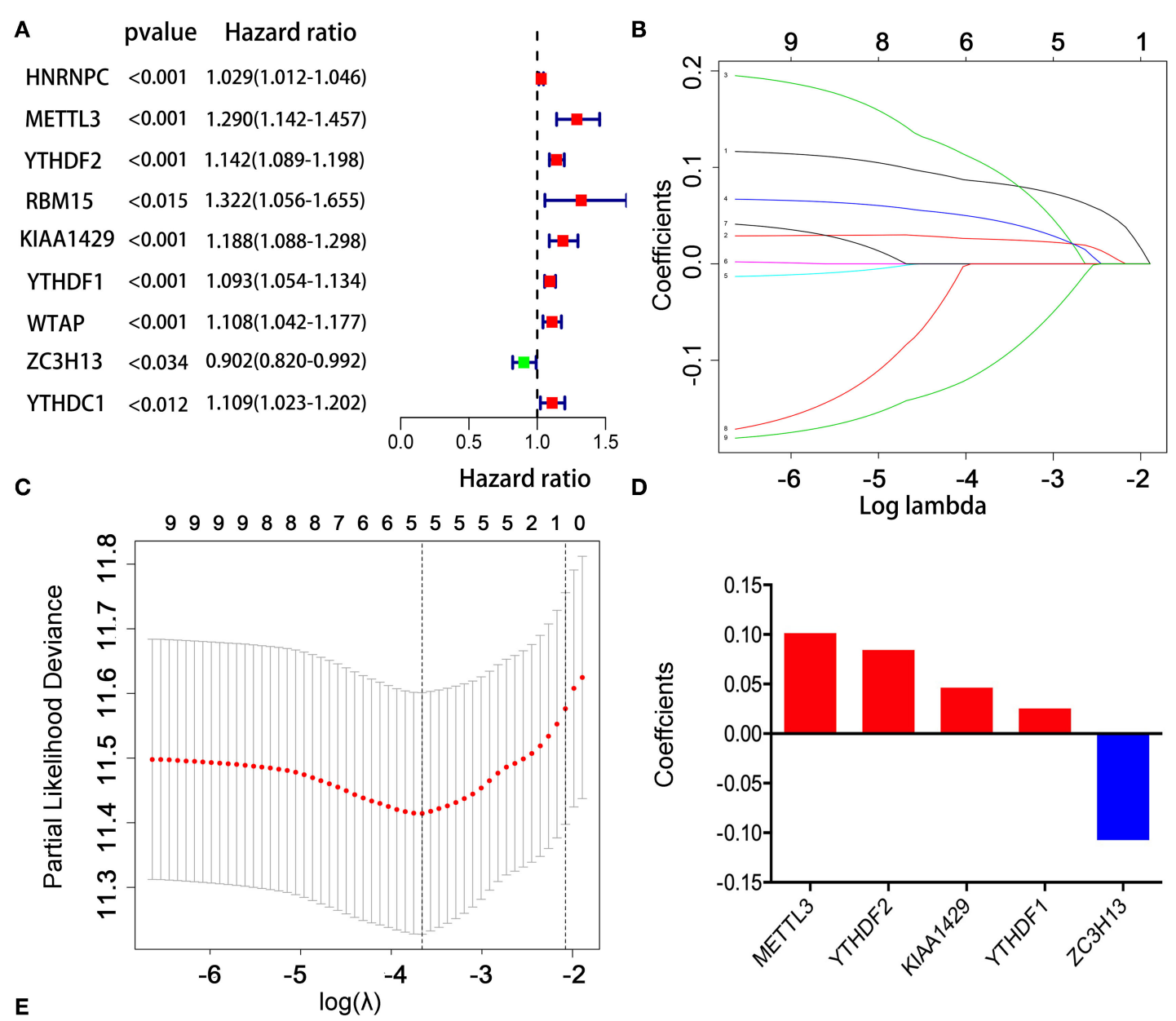

$\mathbf{E}$

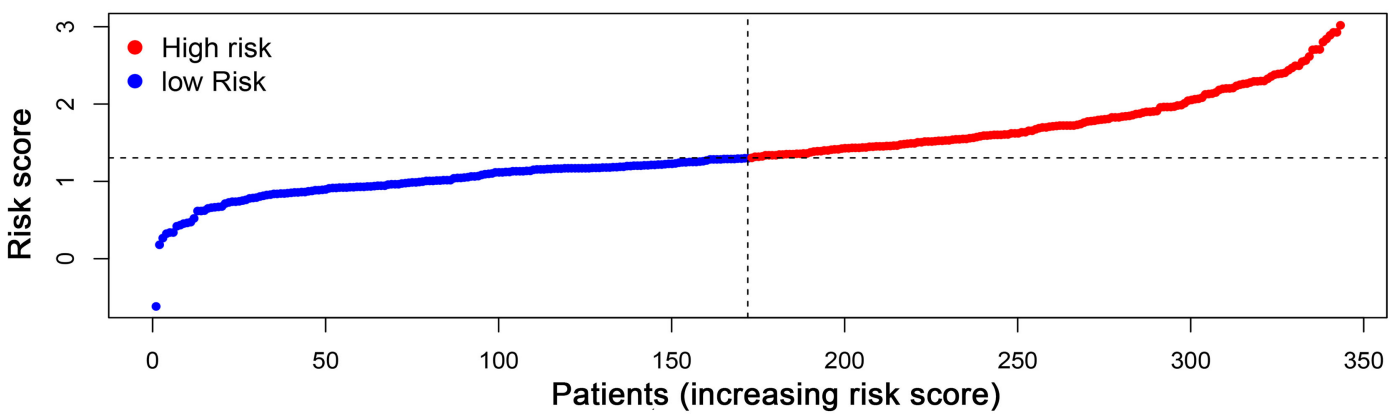

$\mathbf{F}$

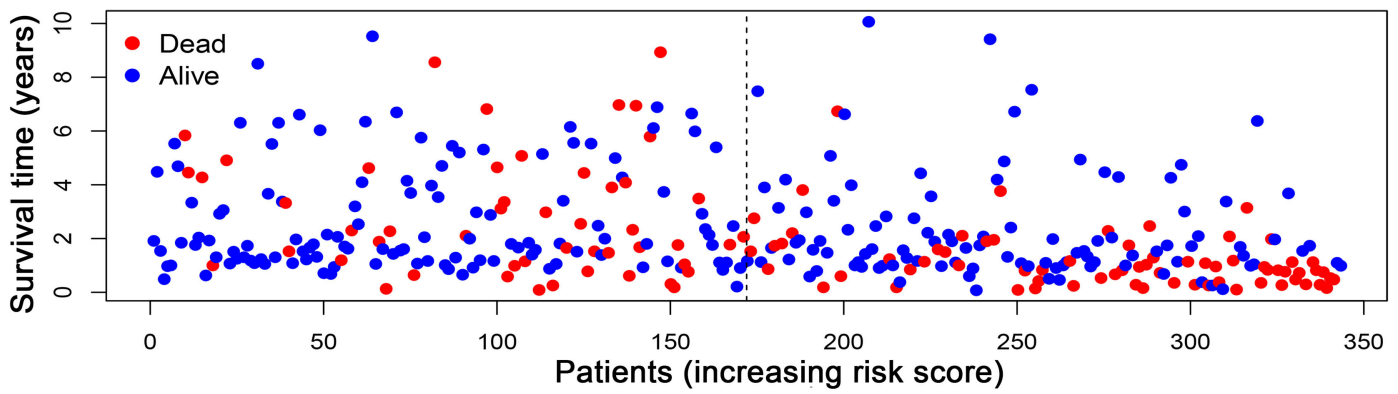

FIGURE 5 | Establishment of the prognostic risk model based on m6A RNA modification regulator genes. (A) Univariate Cox regression analysis of the m6A RNA methylation regulators. (B-D) The process of constructing the signature using absolute shrinkage and selection operator (LASSO) Cox regression. (E) The distributions of risk scores in the prognostic model. (F) The distributions of survival status in the prognostic model. 
demonstrated a worse OS in patients with a high-risk score relative to patients with a low-risk score (Figure 6A, $p=$ $1.118 \mathrm{e}-04)$. The 5 -year OS rate was $43.4 \%$ in the high-risk group and $57.4 \%$ in the low-risk group. We then performed a ROC curve analysis and assessed the area under this curve (AUC) of $0.782,0.723$, and 0.617 for the 1-, 3-, and 5-year OS, respectively, which showed good predictive power for survival outcomes (Figure 6B). Moreover, the risk score distribution of patients with HCC was plotted, as shown in Figure 5E. A dot pot was used to display the survival status of each patient (Figure 5F). The expression of five prognostic genes in the high- and lowrisk groups was displayed in a heatmap (Figure 7A). Clinical relevance was simultaneously plotted above the heatmap. When comparing the clinical parameters between the low- and highrisk groups, significant differences were observed in terms of stage $(p<0.01)$ and grade $(p<0.001)$.

\section{Validation of the Prognostic Signature Using the GEO Database}

To evaluate the prognostic value of the five-gene signature for survival prediction in other datasets, we used the GEO microarray data (GSE54236) for validation (22). A total of 78 HCC patients in the GSE54236 cohort were divided into highrisk $(n=42)$ and low-risk $(n=36)$ groups according to the cutoff value of the TCGA cohort. Same as the results in the TCGA cohort, the survival analysis demonstrated that HCC patients in the low-risk group had markedly better OS compared to high-risk patients (Figure 6C, $\mathrm{p}=5.811 \mathrm{e}-4$ ). The AUCs for $1-, 2-$, and 3 -year OS were $0.689,0.705$, and 0.696 , respectively, indicating that this prognostic model could predict OS of HCC patients with a good accuracy (Figure 6D). Since there was no patient survival beyond 5 years, the 5 -year ROC curve was not plotted.
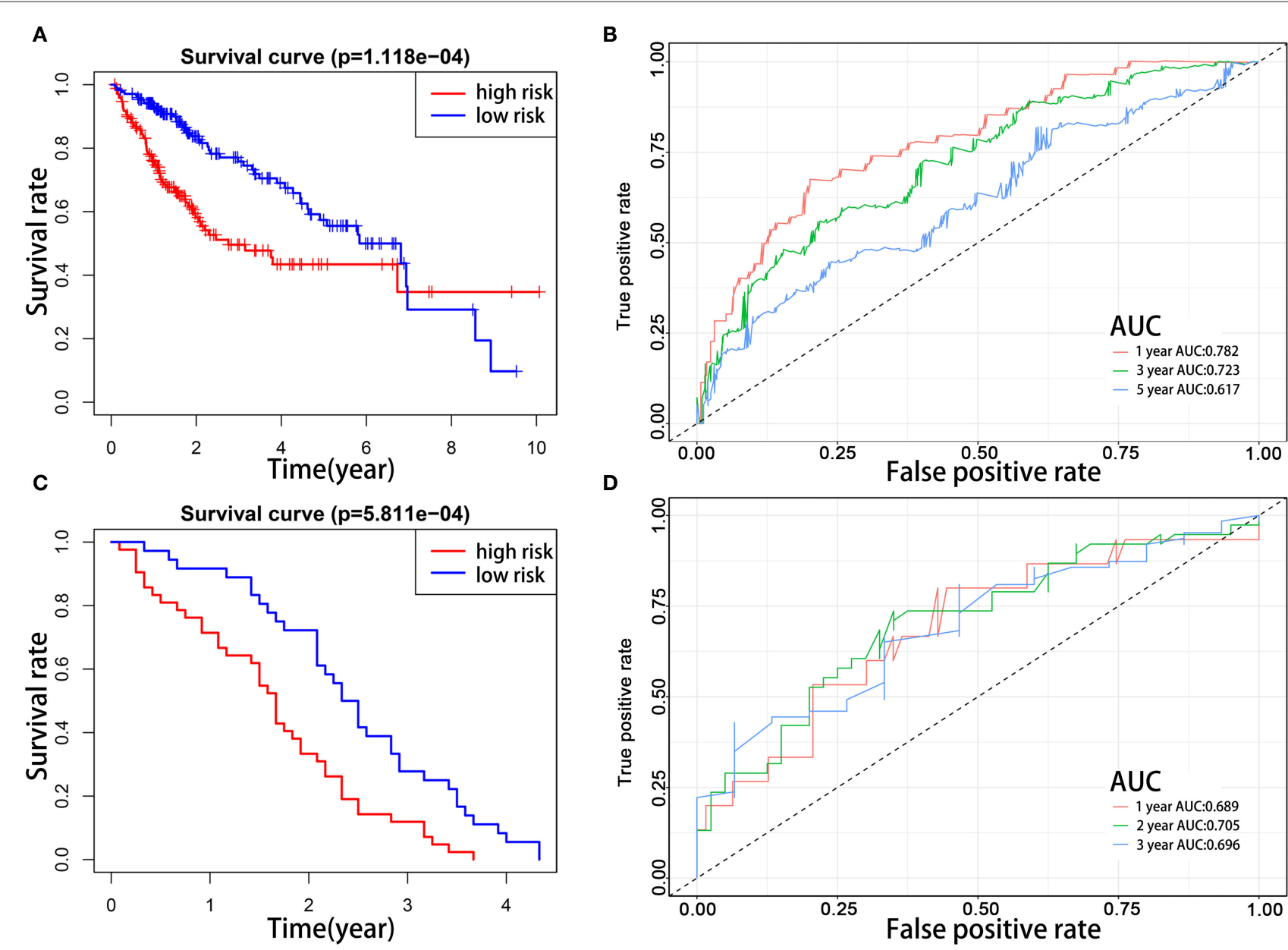

FIGURE 6 | Kaplan-Meier survival analyses of prognostic model. Patients in two datasets were assigned to low-risk (blue) and high-risk (red) groups using median risk score as the cutoff. (A,B) In the TCGA cohort, the survival probability of the low-risk group is higher than the high-risk group ( $p=1.118 \mathrm{e}-4)$. The 1-, 3-, and 5-year AUCs were $0.782,0.723$, and 0.617, respectively. (C,D) The prognostic model was validated in the GEO cohort. The survival probabilities were higher for the low-risk group than the high-risk group ( $p=5.811$-04). The 1-, 2-, and 3-year AUCs were 0.689, 0.705, and 0.696, respectively. 


\section{The Five-Gene Risk Signature Independently Predicts the Prognostic of HCC Patients}

After excluding cases with incomplete clinical information, 339 cases were eligible for Cox regression analysis. Univariate analysis revealed that the five-gene risk score and stage were significantly related to the OS of patients with HCC (Figure 7B, $p$ $<0.001)$. In order to evaluate whether the five-gene risk signature was independent from other clinicopathologic characteristics as a prognostic factor for HCC, we additionally conducted multivariate Cox regression analyses, which demonstrated that both risk score and stage were independently correlated with OS for patients with HCC (Figure 7C, $p<0.001$ ). These results demonstrated that the five-gene risk signature was able to predict prognosis independently of gender, age, histological grade and pathological stage, indicating that this five-gene risk signature could serve as an independent prognostic factor for HCC.

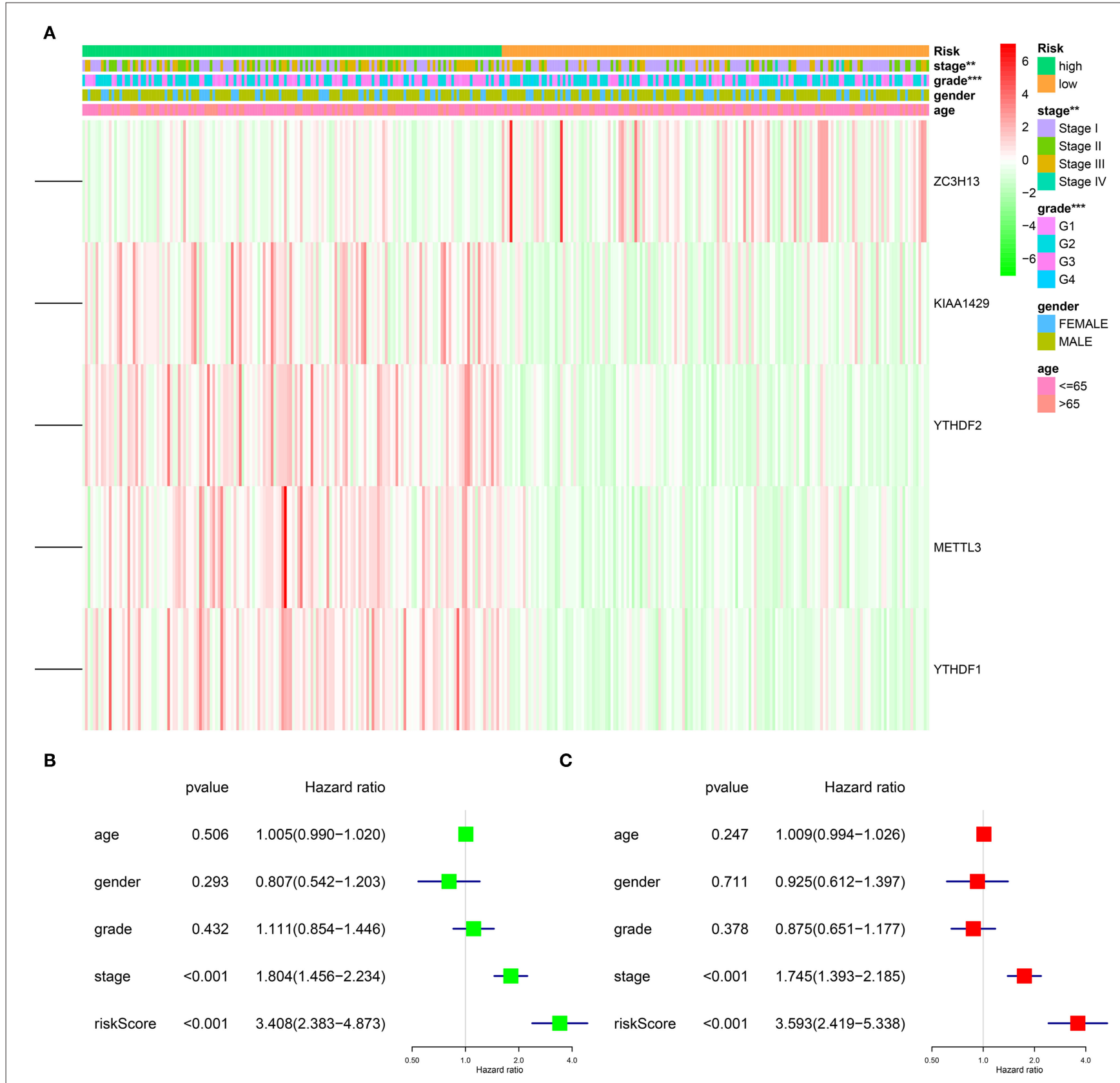

FIGURE 7 | Impact of risk score and clinicopathological features on the prognosis of HCC patients. (A) Heatmap shows the distribution of clinicopathological features and the expression of five m6A RNA modification regulators in high- and low-risk groups. (B) Univariate Cox regression analyses of clinicopathological parameters and OS. (C) Multivariate Cox regression analyses of clinicopathological parameters and OS. ${ }^{* *} p<0.01,{ }^{* * *} p<0.001$. 


\section{Establishment of a Prognostic Nomogram for HCC}

To provide a quantitative method to predict the survival of individuals, we established a novel prognostic nomogram on the basis of age, gender, histological grade, pathological stage, and risk score (Figure 8). The results showed that the nomogram could systematically predict the 1-, 3-, and 5-year OS of patients with HCC.

\section{DISCUSSION}

Globally, HCC is the most prevalent type of liver malignancy, which ranks as the fourth of cancer mortality (1). However, there is currently no effective therapy, and the OS of patients with HCC is still far from satisfactory. Therefore, it is urgent to elucidate the underlying molecular mechanisms contributing to tumorigenesis in HCC. RNA m6A modification, as a new dimension of gene expression control, has aroused strong interest among the academic community in recent years. However, the study of m6A modification in the cancer field is still in its initial stage. Due to the wide application of RNA-seq and microarray techniques, risk scoring systems based on multiplegene signature are increasingly frequently applied to predict prognosis for human cancers (23-25). In the present study, we established a prognostic signature using five m6A RNA modification regulators. Encouragingly, the risk score was able to independently predict the prognosis of HCC patients. Therefore, the risk signature in this study can help clinicians perform individualized survival predictions more accurately.

As shown in our prognostic model, $\mathrm{ZC} 3 \mathrm{H} 13$ was the only m6A-related gene positively associated with HCC patient prognosis, indicating that $\mathrm{ZC} 3 \mathrm{H} 13$ might exert a suppressive effect on HCC. ZC3H13 encodes a CCCH-type zinc finger protein, which plays an important role in the modulation of RNA m6A modification in the nucleus $(26,27)$. To date, even though a few studies have reported the correlation between ZC3H13 and human tumors, its biological function still needs further investigation. According to published studies, $\mathrm{ZC} 3 \mathrm{H} 13$ exhibited heterogeneous roles in various types of human cancer. For example, Zhu et al. found that ZC3H13 inhibits colorectal cancer (CRC) proliferation and invasion via inactivating RasERK signaling pathway, suggesting that $\mathrm{ZC} 3 \mathrm{H} 13$ acts as a tumor suppressor in CRC (28). Similarly, Kim et al. demonstrated that $\mathrm{ZC} 3 \mathrm{H} 13$ is often mutated in $\mathrm{CRC}$, suggesting that $\mathrm{ZC} 3 \mathrm{H} 13$ might function as a tumor suppressor (26). On the contrary, Gewurz et al. revealed that $\mathrm{ZC} 3 \mathrm{H} 13$ acts as a key upstream regulator of the NFKB activation pathway. Since hyperactivation of the $\mathrm{NF} \kappa \mathrm{B}$ promotes tumor proliferation and invasion, $\mathrm{ZC} 3 \mathrm{H} 13$ might function as an oncogene (29). Therefore, the specific role of $\mathrm{ZC} 3 \mathrm{H} 13$ in $\mathrm{HCC}$ remains elusive, which requires indepth research.

Our prognostic model showed that the expression of the remaining four genes (YTHDF1, YTHDF2, METTL3, and KIAA1429) was adversely associated with the prognosis of patients with HCC. Chen et al. reported that METTL3 is significantly upregulated in HCC. Overexpression of METTL3

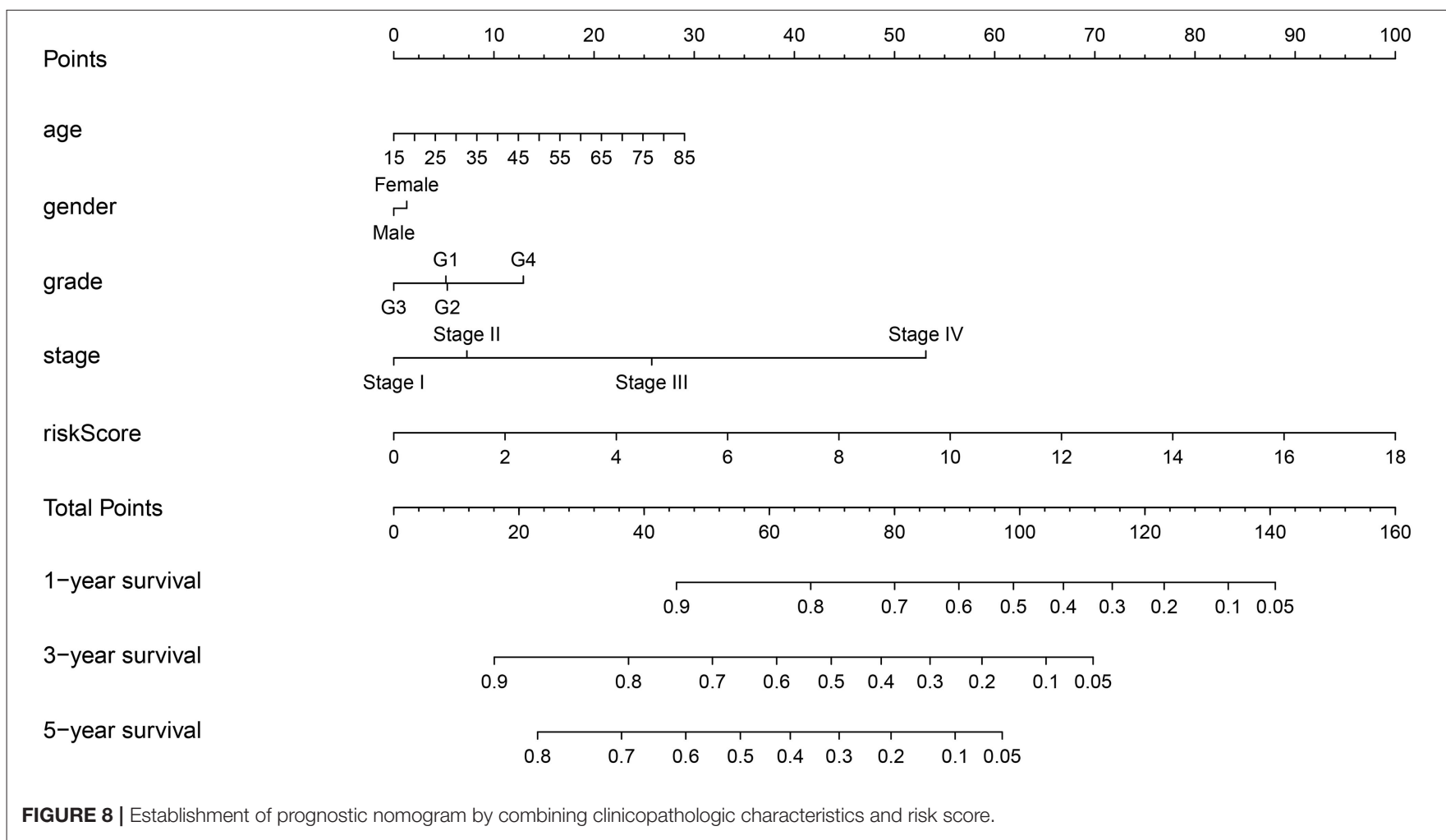


in HCC is linked to poor prognosis. In addition, knockdown of METTL3 impairs HCC oncogenicity and lung metastasis, indicating that METTL3 might act as an oncogene in HCC. Mechanistically, METTL3 promotes the progression of HCC through post-transcriptional silencing SOCS2 (a tumor suppressor gene) in a YTHDF2-dependent manner (15). Vice versa, Lin et al. revealed that the suppression of METTL3 attenuated EMT through downregulating the translation of Snail (16). In agreement with the oncogenic functions of METTL3 in HCC, similar observations have recently been reported in several other types of cancer, namely, AML (30), GMB (31), bladder cancer (32), gastric cancer (33), and breast cancer (34).

KIAA1429, a relatively new component of the m6A "writer" complex, has also been reported to be upregulated in HCC (35). Clinically, overexpression of KIAA1429 is linked to a worse prognosis for HCC patients. Mechanistically, KIAA1429 promotes HCC progression by inhibiting ID2 via upregulating its m6A level (35). Similarly, Lan et al. also showed that KIAA1429 is highly expressed in HCC and correlated with poor prognosis of HCC patients. Their study demonstrated that silencing KIAA1429 inhibited proliferation and metastasis of cancer cells (36). Mechanistically, KIAA1429 could induce m6A modification of GATA3 pre-mRNA, resulting in the degradation of GATA3 pre-mRNA, thus contributing to HCC progression. Interestingly, Qian et al. reported that KIAA1429 could exert oncogenic functions in breast cancer through positive regulating CDK1 in an m6A-independent manner (37). These studies suggest that KIAA1429 is an oncogenic protein that functions through multiple signaling pathways.

YTHDF1 and YTHDF2 are both members of the YTH domain family, which also includes YTHDF3, YTHDC1, and YTHDC2. YTHDF1 is an m6A "reader," which recognizes and binds to $\mathrm{m}^{6} \mathrm{~A}$-modified mRNA, thus improving the translation efficiency of their target RNAs (38). In a recent study, YTHDF1 was reported to be upregulated in HCC, and its overexpression is highly related to unfavorable prognosis (39), which is consistent with the observations of Zhao et al. (40). Additionally, it has been reported that YTHDF1 is highly expressed in CRC and serves as an oncogene in CRC via promoting CRC cell oncogenesis and stem cell-like activity through the Wnt/ $\beta$-catenin pathway $(41,42)$.

According to current research, the major function of YTHDF2 is to regulate mRNA degradation (43). Yang et al. found that YTHDF2 is upregulated in HCC, and miR145 is a negative posttranscriptional regulator of YTHDF2. Also, miR145 downregulates the expression of YTHDF2 through elevating m6A levels via binding to the $3^{\prime}$ UTR of YTHDF2 mRNA (14). On the contrary, Hou et al. revealed that YTHDF2 was downregulated in HCC. YTHDF2 deficiency robustly facilitated HCC growth and metastasis, suggesting that YTHDF2 is a tumor suppressor in HCC. Mechanistically, Hou et al. showed that YTHDF2 inhibited cancer progression by promoting the decay of IL11 and SERPINE2 mRNAs (44). Therefore, the specific role of YTHDF2 in HCC remains controversial. These contradictory findings may be due to the high heterogeneity of HCC. Further studies are required to address these conflicting observations.
Some studies have reported that deregulation of $\mathrm{m} 6 \mathrm{~A}$ regulators is associated with the drug resistance in tumors. Therefore, it is critical to know the expression of m6A regulators in cancer patients in order to choose individualized chemotherapeutic regimens. METTL3 is known to promote the resistance of pancreatic cancer cells against chemotherapy and radiotherapy. Knockdown of METTL3 can significantly increase the sensitivity of pancreatic cancer cells to 5-fluorouracil (5FU), cisplatin, gemcitabine, and radiotherapy (45). Similarly, METTL3 is overexpressed in gliomas and is involved in the maintenance of its radio-resistance (31). In colorectal cancer, knockout of YTHDF1 can suppress the proliferation of cancer cells and enhance their sensitivity to chemotherapy drugs such as 5-FU and oxaliplatin (42). FTO is highly expressed in cervical squamous cell carcinoma (CSCC) tissues. By activating $\beta$-catenin and excision repair pathways, FTO enhances the chemo-radiotherapy resistance of CSCC (46). In tyrosine kinase inhibitor (TKI)-resistant leukemia cells, decreased m6A levels by FTO upregulation results in the overexpression of survival and proliferation-related genes. Vice versa, knockdown of FTO rendered resistant leukemia cells remarkably sensitive to TKI treatments (47). These studies highlight the significance of $\mathrm{m} 6 \mathrm{~A}$ in chemo-radiotherapy resistance and suggest its potential value as a treatment target. At present, there are no studies investigating the role of $\mathrm{m} 6 \mathrm{~A}$ in drug resistance in HCC. Nevertheless, given that METTL3 and YTHDF1 are significantly associated with prognosis of HCC patients, elucidating the mechanisms of m6A in HCC chemoresistance is of great importance for the treatment of drug resistance in HCC.

Previous studies have shown that m6A plays an important role in tumor initiation, progression, metastasis, and other malignant biological behaviors. Therefore, the development of specific inhibitors of $\mathrm{m} 6 \mathrm{~A}$ regulators has great scientific significance and clinical value. Rhein is the first FTO inhibitor that exerts its inhibitory effect by competitive binding to the FTO active site (48). However, rhein has the problem of low specificity due to its cross-activity with ALKB family demethylases (49). Meclofenamic acid (MA) is a highly selective FTO inhibitor that can bind to FTO and stabilize FTO without affecting the demethylase activity of ALKBH5 (50). In addition to natural products, Huang et al. developed two FTO inhibitors, namely, FB23 and FB23-2, by structure-based rational design. In vitro and in vivo experimental evidence demonstrated that FB23-2 exhibits a potent ability to suppress the progression of AML (51). More recently, based on structure-based virtual screening and a series of in vivo and in vitro experiments, Peng et al. discovered that entacapone, which was previously approved for the treatment of Parkinson's disease by the Food and Drug Administration (FDA), can be used as a specific inhibitor of FTO. Entacapone inhibits FTO activity through competitive binding with m6Amodified RNA substrates. After treatment with entacapone, the diet-induced obese mice showed a significant decrease in body weight and blood glucose levels (52). Science entacapone is already an FDA-approved drug, and it could be readily generalizable to other clinical indications that are related to FTO, such as cancer and obesity. For the time being, other than 
FTO, there are no known inhibitors for m6A regulatory. More effective and specific inhibitors for targeting $\mathrm{m} 6 \mathrm{~A}$ regulatory are urgently needed. The development of such inhibitors will not only deepen the understanding about the mechanism of $\mathrm{m} 6 \mathrm{~A}$ in carcinogenesis but also provide more tools for designing novel therapies.

Nevertheless, we acknowledge that several limitations in this study deserve mention. First, since our data are drawn from the TCGA and GEO databases, further experimental evidence is needed to verify our findings. Second, the sample size varied significantly between the normal and tumor groups, which may affect the reliability of the results. Finally, as the main patients are Americans and Italians, selection bias inevitably occurred. As a result, the findings in our study might not be generalizable to all populations.

In summary, we demonstrated that the gene expression signature of m6A modification regulators possesses great potential for HCC prognosis prediction. Our study offers additional evidence for further research regarding m6A RNA modification in HCC. However, further experimental and clinical exploration are necessary to confirm these findings.

\section{REFERENCES}

1. Bray F, Ferlay J, Soerjomataram I, Siegel RL, Torre LA, Jemal A. Global cancer statistics 2018: GLOBOCAN estimates of incidence and mortality worldwide for 36 cancers in 185 countries. CA Cancer J Clin. (2018) 68:394-424. doi: $10.3322 /$ caac. 21492

2. El-Serag HB, Rudolph KL. Hepatocellular carcinoma: epidemiology and molecular carcinogenesis. Gastroenterology. (2007) 132:2557-76. doi: 10.1053/j.gastro.2007.04.061

3. Frenette C, Gish RG. Hepatocellular carcinoma: molecular and genomic guideline for the clinician. Clin Liver Dis. (2011) 15:307-21, vii-x. doi: 10.1016/j.cld.2011.03.010

4. Roessler S, Jia HL, Budhu A, Forgues M, Ye QH, Lee JS, et al. A unique metastasis gene signature enables prediction of tumor relapse in earlystage hepatocellular carcinoma patients. Cancer Res. (2010) 70:10202-12. doi: 10.1158/0008-5472.CAN-10-2607

5. Krug RM, Morgan MA, Shatkin AJ. Influenza viral mRNA contains internal N6-methyladenosine and 5'-terminal 7-methylguanosine in cap structures. J Virol. (1976) 20:45-53. doi: 10.1128/JVI.20.1.45-53.1976

6. Horowitz S, Horowitz A, Nilsen TW, Munns TW, Rottman FM. Mapping of N6-methyladenosine residues in bovine prolactin mRNA. Proc Natl Acad Sci USA. (1984) 81:5667-71. doi: 10.1073/pnas.81.18.5667

7. Yang Y, Hsu PJ, Chen YS, Yang YG. Dynamic transcriptomic m(6)A decoration: writers, erasers, readers and functions in RNA metabolism. Cell Res. (2018) 28:616-24. doi: 10.1038/s41422-018-0040-8

8. Tuncel G, Kalkan R. Importance of $m$ N(6)-methyladenosine. (m(6)A) RNA modification in cancer. Med Oncol. (2019) 36:36. doi: 10.1007/s12032-019-1260-6

9. Jia G, Fu Y, Zhao X, Dai Q, Zheng G, Yang Y, et al. N6-methyladenosine in nuclear RNA is a major substrate of the obesity-associated FTO. Nat Chem Biol. (2011) 7:885-7. doi: 10.1038/nchembio.687

10. Zheng G, Dahl JA, Niu Y, Fedorcsak P, Huang CM, Li CJ, et al. ALKBH5 is a mammalian RNA demethylase that impacts RNA metabolism and mouse fertility. Mol Cell. (2013) 49:18-29. doi: 10.1016/j.molcel.2012.10.015

11. Zhang C, Fu J, Zhou Y. A review in research progress concerning m6A methylation and immunoregulation. Front Immunol. (2019) 10:922. doi: $10.3389 /$ fimmu.2019.00922

\section{DATA AVAILABILITY STATEMENT}

All datasets presented in this study are included in the article/supplementary material.

\section{AUTHOR CONTRIBUTIONS}

SL, SZ, and LZha designed the study. LZha and YQ collected the data. JH, LZho, and DW performed the bioinformatics analysis. LZha and YQ wrote the manuscript. SL and SZ were responsible for the supervision of the work. All authors contributed to the article and approved the submitted version.

\section{FUNDING}

Funding was provided by the Innovative Research Groups of the National Natural Science Foundation of China (No. 81721091), the National S\&T Major Project (No. 2017ZX10203205), the Zhejiang International Science and Technology Cooperation Project (No. 2016C04003), the Research Unit Project of the Chinese Academy of Medical Sciences (2019-I2M-5-030), and the Major Program of the National Natural Science Foundation of China (No. 91542205).

12. Sun $\mathrm{T}, \mathrm{Wu} \mathrm{R}$, Ming L. The role of m6A RNA methylation in cancer. Biomed Pharmacother. (2019) 112:108613. doi: 10.1016/j.biopha.2019.108613

13. Ma JZ, Yang F, Zhou CC, Liu F, Yuan JH, Wang F, et al. METTL14 suppresses the metastatic potential of hepatocellular carcinoma by modulating $\mathrm{N}(6)$ -methyladenosine-dependent primary MicroRNA processing. Hepatology. (2017) 65:529-43. doi: 10.1002/hep.28885

14. Yang Z, Li J, Feng G, Gao S, Wang Y, Zhang S, et al. MicroRNA-145 modulates $\mathrm{N}(6)$-methyladenosine levels by targeting the 3'-untranslated mRNA region of the N(6)-methyladenosine binding YTH domain family 2 protein. J Biol Chem. (2017) 292:3614-23. doi: 10.1074/jbc.M116.749689

15. Chen M, Wei L, Law CT, Tsang FH, Shen J, Cheng CL, et al. RNA N6-methyladenosine methyltransferase-like 3 promotes liver cancer progression through YTHDF2-dependent posttranscriptional silencing of SOCS2. Hepatology. (2018) 67:2254-70. doi: 10.1002/hep.29683

16. Lin X, Chai G, Wu Y, Li J, Chen F, Liu J, et al. RNA m(6)A methylation regulates the epithelial mesenchymal transition of cancer cells and translation of Snail. Nat Commun. (2019) 10:2065. doi: 10.1038/s41467-019-09865-9

17. Zhang C, Samanta D, Lu H, Bullen JW, Zhang H, Chen I, et al. Hypoxia induces the breast cancer stem cell phenotype by HIF-dependent and ALKBH5-mediated m(6)A-demethylation of NANOG mRNA. Proc Natl Acad Sci USA. (2016) 113:E2047-56. doi: 10.1073/pnas.1602883113

18. Panneerdoss S, Eedunuri VK, Yadav P, Timilsina S, Rajamanickam S, Viswanadhapalli S, et al. Cross-talk among writers, readers, and erasers of m(6)A regulates cancer growth and progression. Sci Adv. (2018) 4:eaar8263. doi: 10.1126/sciadv.aar8263

19. Chen M, Wong CM. The emerging roles of N6-methyladenosine (m6A) deregulation in liver carcinogenesis. Mol Cancer. (2020) 19:44. doi: 10.1186/s12943-020-01172-y

20. Sheng H, Li Z, Su S, Sun W, Zhang X, Li L, et al. YTH domain family 2 promotes lung cancer cell growth by facilitating 6-phosphogluconate dehydrogenase mRNA translation. Carcinogenesis. (2020) 41:541-50. doi: 10.1093/carcin/bgz152

21. Wang X, Wu R, Liu Y, Zhao Y, Bi Z, Yao Y, et al. m(6)A mRNA methylation controls autophagy and adipogenesis by targeting Atg5 and Atg7. Autophagy. (2020) 16:1221-35. doi: 10.1080/15548627.2019.1659617

22. Villa E, Critelli R, Lei B, Marzocchi G, Camma C, Giannelli G, et al. Neoangiogenesis-related genes are hallmarks of fast-growing hepatocellular 
carcinomas and worst survival. Results from a prospective study. Gut. (2016) 65:861-9. doi: 10.1136/gutjnl-2014-308483

23. Chen HY, Yu SL, Chen CH, Chang GC, Chen CY, Yuan A, et al. A five-gene signature and clinical outcome in non-small-cell lung cancer. $N$ Engl J Med. (2007) 356:11-20. doi: 10.1056/NEJMoa060096

24. Zhang JX, Song W, Chen ZH, Wei JH, Liao YJ, Lei J, et al. Prognostic and predictive value of a microRNA signature in stage II colon cancer: a microRNA expression analysis. Lancet Oncol. (2013) 14:1295-306. doi: 10.1016/S1470-2045(13)70491-1

25. Wang Y, Zhang Q, Gao Z, Xin S, Zhao Y, Zhang K, et al. A novel 4-gene signature for overall survival prediction in lung adenocarcinoma patients with lymph node metastasis. Cancer Cell Int. (2019) 19:100. doi: 10.1186/s12935-019-0822-1

26. Kim YR, Chung NG, Kang MR, Yoo NJ, Lee SH. Novel somatic frameshift mutations of genes related to cell cycle and DNA damage response in gastric and colorectal cancers with microsatellite instability. Tumori. (2010) 96:1004-9.

27. Wen J, Lv R, Ma H, Shen $\mathrm{H}, \mathrm{He} \mathrm{C}$, Wang J, et al. Zc3h13 regulates nuclear RNA m(6)A methylation and mouse embryonic stem cell self-renewal. Mol Cell. (2018) 69:1028-38 e1026. doi: 10.1016/j.molcel.2018.02.015

28. Zhu D, Zhou J, Zhao J, Jiang G, Zhang X, Zhang Y, et al. ZC3H13 suppresses colorectal cancer proliferation and invasion via inactivating RasERK signaling. J Cell Physiol. (2019) 234:8899-907. doi: 10.1002/jcp.27551

29. Gewurz BE, Towfic F, Mar JC, Shinners NP, Takasaki K, Zhao B, et al. Genomewide siRNA screen for mediators of NF-kappaB activation. Proc Natl Acad Sci USA. (2012) 109:2467-72. doi: 10.1073/pnas.1120542109

30. Vu LP, Pickering BF, Cheng Y, Zaccara S, Nguyen D, Minuesa G, et al. The $\mathrm{N}(6)$-methyladenosine (m(6)A)-forming enzyme METTL3 controls myeloid differentiation of normal hematopoietic and leukemia cells. Nat Med. (2017) 23:1369-76. doi: 10.1038/nm.4416

31. Visvanathan A, Patil V, Arora A, Hegde AS, Arivazhagan A, Santosh V, et al. Essential role of METTL3-mediated m(6)A modification in glioma stem-like cells maintenance and radioresistance. Oncogene. (2018) 37:522-33. doi: 10.1038/onc.2017.351

32. Cheng $\mathrm{M}$, Sheng L, Gao Q, Xiong Q, Zhang $\mathrm{H}$, Wu M, et al. The m(6)A methyltransferase METTL3 promotes bladder cancer progression via AFF4/NF-kappaB/MYC signaling network. Oncogene. (2019) 38:3667-80. doi: 10.1038/s41388-019-0683-z

33. Liu T, Yang S, Sui J, Xu SY, Cheng YP, Shen B, et al. Dysregulated N6methyladenosine methylation writer METTL3 contributes to the proliferation and migration of gastric cancer. J Cell Physiol. (2020) 235:548-62. doi: $10.1002 /$ jcp. 28994

34. Cai X, Wang X, Cao C, Gao Y, Zhang S, Yang Z, et al. HBXIPelevated methyltransferase METTL3 promotes the progression of breast cancer via inhibiting tumor suppressor let-7g. Cancer Lett. (2018) 415:11-9. doi: 10.1016/j.canlet.2017.11.018

35. Cheng X, Li M, Rao X, Zhang W, Li X, Wang L, et al. KIAA1429 regulates the migration and invasion of hepatocellular carcinoma by altering m6A modification of ID2 mRNA. Onco Targets Ther. (2019) 12:3421-8. doi: 10.2147/OTT.S180954

36. Lan $\mathrm{T}$, Li H, Zhang $\mathrm{D}$, Xu L, Liu H, Hao X, et al. KIAA1429 contributes to liver cancer progression through N6-methyladenosinedependent post-transcriptional modification of GATA3. Mol Cancer. (2019) 18:186. doi: 10.1186/s12943-019-1106-Z

37. Qian JY, Gao J, Sun X, Cao MD, Shi L, Xia TS, et al. KIAA1429 acts as an oncogenic factor in breast cancer by regulating CDK1 in an N6-methyladenosine-independent manner. Oncogene. (2019) 38:6123-41. doi: 10.1038/s41388-019-0861-Z

38. Wang X, Zhao BS, Roundtree IA, Lu Z, Han D, Ma H, et al. N(6)methyladenosine Modulates Messenger RNA Translation Efficiency. Cell. (2015) 161:1388-99. doi: 10.1016/j.cell.2015.05.014
39. Zhou Y, Yin Z, Hou B, Yu M, Chen R, Jin H, et al. Expression profiles and prognostic significance of RNA N6-methyladenosine-related genes in patients with hepatocellular carcinoma: evidence from independent datasets. Cancer Manag Res. (2019) 11:3921-31. doi: 10.2147/CMAR.S191565

40. Zhao X, Chen Y, Mao Q, Jiang X, Jiang W, Chen J, et al. Overexpression of YTHDF1 is associated with poor prognosis in patients with hepatocellular carcinoma. Cancer Biomark. (2018) 21:859-68. doi: 10.3233/CBM-1 70791

41. Bai Y, Yang C, Wu R, Huang L, Song S, Li W, et al. YTHDF1 regulates tumorigenicity and cancer stem cell-like activity in human colorectal carcinoma. Front Oncol. (2019) 9:332. doi: 10.3389/fonc.2019.00332

42. Nishizawa Y, Konno M, Asai A, Koseki J, Kawamoto K, Miyoshi $\mathrm{N}$, et al. Oncogene c-Myc promotes epitranscriptome $\mathrm{m}(6) \mathrm{A}$ reader YTHDF1 expression in colorectal cancer. Oncotarget. (2018) 9:7476-86. doi: 10.18632/oncotarget.23554

43. Du H, Zhao Y, He J, Zhang Y, Xi H, Liu M, et al. YTHDF2 destabilizes m(6)Acontaining RNA through direct recruitment of the CCR4-NOT deadenylase complex. Nat Commun. (2016) 7:12626. doi: 10.1038/ncomms12626

44. Hou J, Zhang H, Liu J, Zhao Z, Wang J, Lu Z, et al. YTHDF2 reduction fuels inflammation and vascular abnormalization in hepatocellular carcinoma. Mol Cancer. (2019) 18:163. doi: 10.1186/s12943-019-1082-3

45. Taketo K, Konno M, Asai A, Koseki J, Toratani M, Satoh T, et al. The epitranscriptome m6A writer METTL3 promotes chemo- and radioresistance in pancreatic cancer cells. Int J Oncol. (2018) 52:621-9. doi: 10.3892/ijo.2017.4219

46. Zhou S, Bai ZL, Xia D, Zhao ZJ, Zhao R, Wang YY, et al. FTO regulates the chemo-radiotherapy resistance of cervical squamous cell carcinoma (CSCC) by targeting beta-catenin through mRNA demethylation. Mol Carcinog. (2018) 57:590-7. doi: 10.1002/mc.22782

47. Yan F, Al-Kali A, Zhang Z, Liu J, Pang J, Zhao N, et al. A dynamic $\mathrm{N}(6)$-methyladenosine methylome regulates intrinsic and acquired resistance to tyrosine kinase inhibitors. Cell Res. (2018) 28:1062-76. doi: 10.1038/s41422-018-0097-4

48. Chen B, Ye F, Yu L, Jia G, Huang X, Zhang X, et al. Development of cell-active N6-methyladenosine RNA demethylase FTO inhibitor. J Am Chem Soc. (2012) 134:17963-71. doi: 10.1021/ja3064149

49. Li Q, Huang Y, Liu X, Gan J, Chen H, Yang CG. Rhein inhibits AlkB repair enzymes and sensitizes cells to methylated DNA damage. J Biol Chem. (2016) 291:11083-93. doi: 10.1074/jbc.M115.711895

50. Huang Y, Yan J, Li Q, Li J, Gong S, Zhou H, et al. Meclofenamic acid selectively inhibits FTO demethylation of m6A over ALKBH5. Nucleic Acids Res. (2015) 43:373-84. doi: 10.1093/nar/gku1276

51. Huang Y, Su R, Sheng Y, Dong L, Dong Z, Xu H, et al. Small-molecule targeting of oncogenic FTO demethylase in acute myeloid leukemia. Cancer Cell. (2019) 35:677-91 e610. doi: 10.1016/j.ccell.2019.03.006

52. Peng S, Xiao W, Ju D, Sun B, Hou N, Liu Q, et al. Identification of entacapone as a chemical inhibitor of FTO mediating metabolic regulation through FOXO1. Sci Transl Med. (2019) 11:eaau7116. doi: 10.1126/scitranslmed.aau7116

Conflict of Interest: The authors declare that the research was conducted in the absence of any commercial or financial relationships that could be construed as a potential conflict of interest.

Copyright (C) 2020 Zhang, Qiao, Huang, Wan, Zhou, Lin and Zheng. This is an open-access article distributed under the terms of the Creative Commons Attribution License (CC BY). The use, distribution or reproduction in other forums is permitted, provided the original author(s) and the copyright owner(s) are credited and that the original publication in this journal is cited, in accordance with accepted academic practice. No use, distribution or reproduction is permitted which does not comply with these terms. 